\title{
CAVEStudy: an Infrastructure for Computational Steering in Virtual Reality Environments
}

\author{
Luc RENAMBOT ${ }^{1}$ \\ Henri E. BAL ${ }^{1,2}$ \\ Desmond GERMANS ${ }^{2}$ \\ Hans J.W. SPOELDER ${ }^{2}$ \\ ${ }^{1}$ Division of Mathematics and Computer Science \\ ${ }^{2}$ Division of Physics and Astronomy \\ Faculty of Sciences, Vrije Universiteit \\ De Boelelaan 1081, 1081 HV Amsterdam, The Netherlands
}

\begin{abstract}
We present the CAVEStudy system that enables scientists to interactively steer a simulation from a virtual reality (VR) environment. No modification to the source code is necessary. CAVEStudy allows interactive and immersive analysis of a simulation running on a remote computer. We describe three case-studies implemented with CAVEStudy.
\end{abstract}

\section{Introduction}

High-speed networks and high performance graphics open opportunities for completely new types of applications. As a result, the world of scientific computing is moving away from the batch-oriented management to interactive programs. Also, virtual reality (VR) systems are now commercially available, but so far scientists mainly use them for off-line visualization of data sets produced by a simulation program. This simulation runs on a remote supercomputer without any user-control. To become widely used by scientists, virtual reality environments should provide tools to connect, visualize, and control on-going simulations. This gives rise to a number of research questions: interaction, collaboration, and steering in virtual reality become central issues in the design of virtual environments for scientific applications.

Networked virtual environments have rapidly developed over the last few years. Numerous toolkits are now available $[2,11,17,18]$. Each one has some specific features, such as collaboration, portability, and distributed simulations. The most general toolkit, and the one most used, is CAVERNSoft [9]. It provides networking and database functionalities needed in a virtual reality system. Different skeletons of VR application are proposed. The system is open but still requires substantial effort to build new applications.
A related research area is the control of a running simulation, referred to as computational steering [16]. It is defined as interactive control over a simulation during its execution. The scientist can control a set of parameters of the program and react to the current results. Computational steering enhances the productivity of the scientist by giving a problemsolving environment [3]. However, existing systems, such as SCIRun [16] and CSE [21], are used to build new applications or require modifications to the source code of the application, and thus are unsuitable if the source code is unavailable.

In this paper, we describe a system called CAVEStudy, based on the CAVERNSoft toolkit, that allows the scientist to steer a program from a virtual reality system without requiring any modification to the program. It enables an interactive and immersive analysis of simulations running on remote computers. CAVEStudy allows non-experts in VR to couple their simulation to virtual environments.

The scientist models the simulation as a set of input, output, and graphical objects. These objects are the input parameters of the simulation and the data produced. Given such a description, our system generates a wrapper around the simulation to control its execution on a remote computing system, which usually is a supercomputer or a cluster. The data produced by the simulation is then packed and sent to the computer hosting the virtual environment. A proxy for the remote simulation is built to receive the data generated by the simulation. This proxy is plugged into a virtual reality environment, where it updates the objects described by the scientist. CAVERNSoft provides the communication and persistence layer needed by our infrastructure. Our infrastructure consists of a description language, a graphical interface to create description files, a code generator, and a virtual reality environment. Thus, it is possible to visualize and control the program directly in the domain space of the simulation. 
The main contributions of our work are as follows :

- a high-level steering system that does not require modifications to the source code,

- a VR framework to immerse the scientist in the simulation space,

- an experimental evaluation of the steering system and VR framework using various systems, such as an IBM SP2, a 128-node Myrinet cluster computer, and several CAVEs and graphic workstations,

- three real applications (collaborative soccer, laser simulation, molecular dynamics visualization) used as case-studies.

This paper is structured as follows. In Section 2, we survey related research. In Section 3, we introduce our system. Using a small example, we show how it facilitates the coupling of a simulation to a virtual environment. Some applications built with our infrastructure are described in Section 4. Finally, we present our conclusions.

\section{Related work}

Our work is related to a wide and active research area referred to as the Grid, Metacomputing, or the next generation high-performance computing infrastructure $[3,4,5]$. The goal is to provide languages, tools, and environments to create new applications that were not conceivable before, such as world-wide collaboration in virtual reality or realtime data-mining of large data sets. Below, we discuss networked virtual environments and steering systems.

Interactive and collaborative visualization radically change the way scientists use computer systems [3]. With interactive visualization, a user can interact with a program in its visual domain. Distributed collaboration allows multiple users at different geographic locations to cooperate, by interacting in real-time through a shared application $[9,19]$. Several toolkits have been designed to provide networked virtual environments: NPSNET, BrickNet, MR Toolkit, DIVE, Spline, Massive, HLA, Bamboo, Avocado, and CAVERNSoft. They provide functionalities such as communication, shared-state management, collaboration mechanisms, and 3D graphics. In the full paper, we compare our work with these systems.

Many existing applications restrict the interaction to the visualization process (e.g., the direction of view, the zoom factor). A more advanced form of interaction, referred to as computational steering, allows the user to interact with the simulation process. Several systems support steering [13], but they typically provide only low-level interactions and require users to monitor or change the application program's internal variables. We do not address the issue of fine grain steering offered by some systems [6], but we focus on the complete execution of a simulation for a given set of inputs. However, we also examine simulations that produce results during the execution (i.e., iterative methods), shipping the intermediate results as output. In the full paper, we discuss several steering systems including SCIRun, VASE, Magellan, CUMULVS, VIPER and CSE.

Our main conclusion is that no environment provides all the functionalities needed for an interactive and immersive steering environment, given that we do not want to modify the source code. We designed CAVEStudy in that spirit.

\section{CAVEStudy}

Our goal is to build a system that combines the power and the functionalities of computational steering and virtual reality. Such an environment, combining both the control over a simulation and the immersion in the data space, does not exist yet as far as we know.

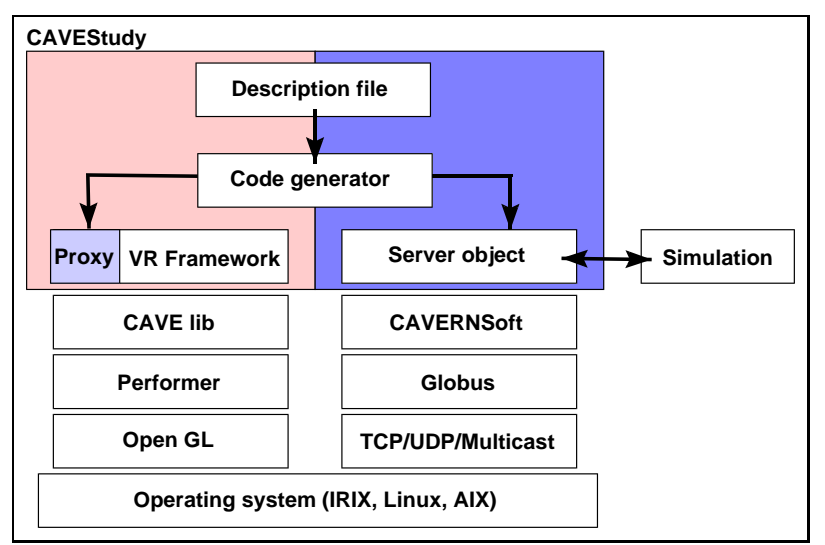

Figure 1. Software layers

CAVEStudy mainly consists of two parts: a code generator and a VR framework, as shown in Figure 1. To minimize the programming for the control of the simulation and the data management, the user has to describe the simulation by a description file. This file is processed to generate two objects, a proxy and a server. The simulation is wrapped into a server object to control its execution. The server's interface provides methods to start, stop, pause, and resume the simulation. The data generated by the simulation are automatically propagated to the proxy object. This object can be seen as a local copy of the remote simulation. Through the network, it reflects the input values and the commands to the server. Furthermore, it manages the incoming data from the simulation and presents them to the VR framework. 


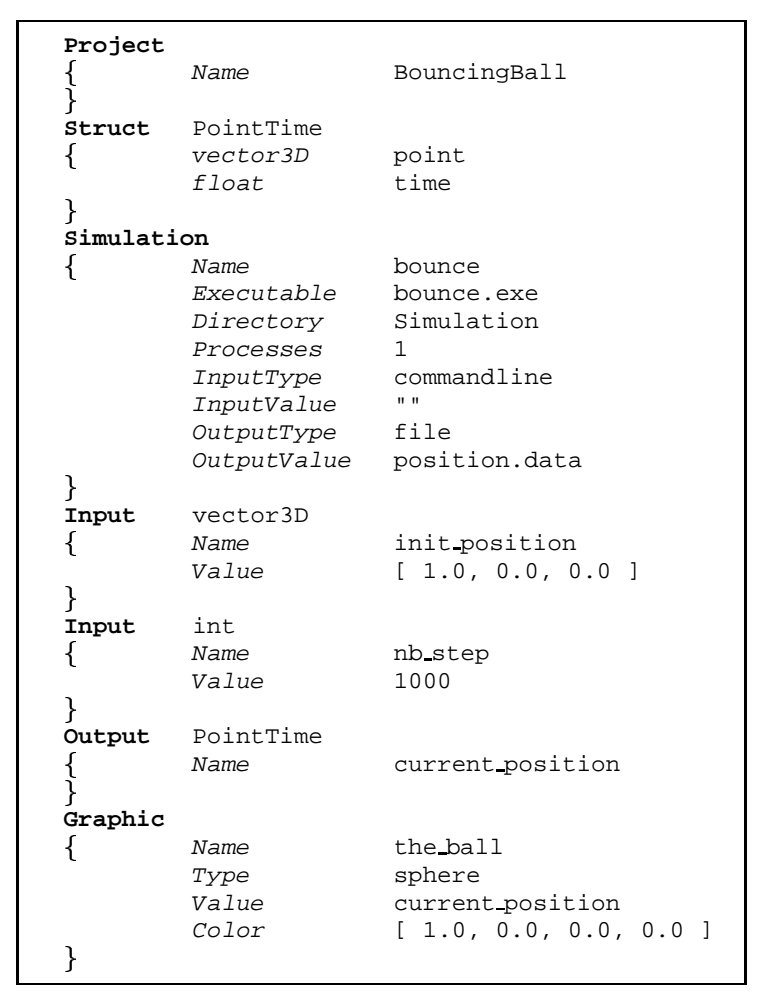

Figure 2. A sample description file

\subsection{Simulation description}

To model a simulation, we designed a description language which allows the user to describe the input parameters and the output data of the simulation. It can be seen as a light-weight and dedicated CORBA-like interface description language. Several sections must be present in such a file: a description of the simulation program, a set of input parameters, and a set of output data. Graphical objects can also be described. An example is presented in Figure 2. A graphical interface is available to describe such a file.

To be able to start the simulation, the user must give information about the executable such as its name and directory. This is specified in the Simulation section. The way to feed the simulation with the input parameters should also be specified, for example as a command-line or with an input file. The system should know how to acquire the output data produced, for instance on the standard output or from a file. Data manipulated by the simulation can be described using either the provided built-in types or by some types defined by the user. The basic types defined in the system are: long, floating-point, string, 2D-point, and 3D-point. New types can be described by combining basic types. An example can be seen in Figure 2 (type PointTime). All these types can be used in scalar, list, and matrix objects.

Input parameters are described by a name, a type, a di- mension (scalar, list, or matrix) and an optional input value when applicable (scalar object). Any number of input parameters can be specified by several Input sections. Output objects are given in a similar way using Output sections. Finally, it is possible to specify graphical objects. Such an object is the graphical representation of an output data produced by the simulation. The user is able to interactively modify these objects, steering visually the simulation. The value of such an object should be an output object, and the type can be selected among several ones (sphere, line, surface, etc).

\subsection{Code generation}

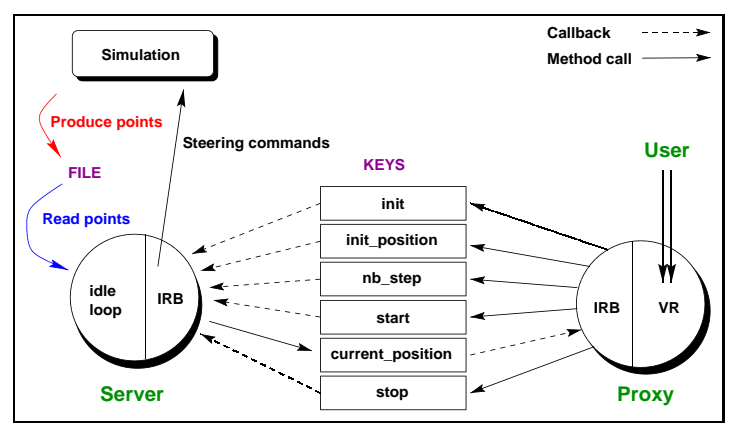

Figure 3. A resulting architecture

We wrote a code generator for the description files. It generates $\mathrm{C}++$ code for the CAVERNSoft [9] network layer, as shown on the right part of Figure 1. We selected CAVERNSoft because it provides functionalities to build networked virtual environments and because it is widely used in the VR community. CAVERNSoft, based on the Nexus communication library from Globus, uses a "publish and subscribe" paradigm. A site can define keys to publish its own data, and a remote site that subscribes to these keys will automatically receive the data through callback functions. This mechanism can be used for small data (tracker data) to large data sets (data-mining) using different policies. CAVERNSoft supports persistence, thread management, and network protocols (TCP/UDP/multicast). It also provides some facilities for avatars and 3D models using the SGI Performer library.

Our code generator produces $\mathrm{C}++$ classes for the server object and the proxy object. Each of these objects contains a threaded IRB (network object of CAVERNSoft) and defines a set of keys with their associated callback functions. For each input or output object, a key is defined to transmit the value. The marshaling code for all the types is generated to be able to use our system in a heterogeneous environment. A set of keys is also created for the control of the simulation (initialize, start, stop, pause, resume, shutdown 
methods). It is therefore possible to manipulate proxy and server entities as $\mathrm{C}++$ objects, without dealing with network issues. Figure 3 presents the resulting architecture corresponding to the description file of Figure 2. It shows the relation between the server object (as referred in Figure 1) and the proxy object. For the server, we generate an program which is basically a endless loop waiting for remote method invocations. The proxy object is embedded into our VR framework.

Performance issues are discussed in the full paper.

\subsection{VR framework}

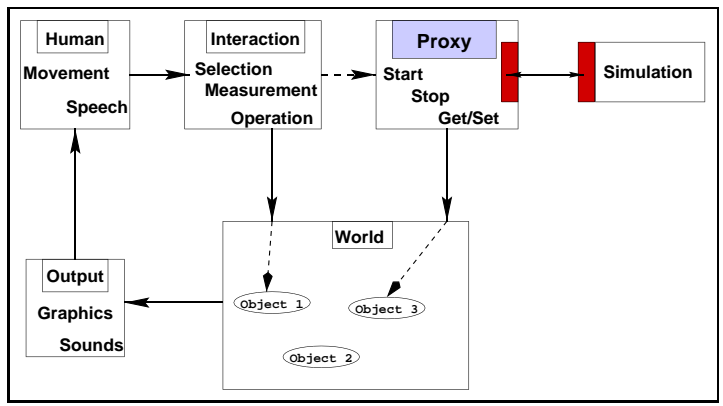

Figure 4. VR framework

Besides the code generator, we developed a virtual reality framework to steer and visualize the data produced by the simulation. It is built on top of the Performer library to exploit the high performance of SGI workstations and on top of the CAVELib library to control VR devices (multiscreens and tracker management), as shown on the left part of Figure 1. The architecture is described in Figure 4, focussing on the VR framework as referred in Figure 1. It consists of a shared-world where the objects of the simulation are represented. These objects are updated through the generated proxy object. An interaction module allows the user to send commands to the proxy or to directly manipulate the objects of the simulation to steer it. This framework is functional but still under development, following the needs of the applications described in the next section.

Figure 5 shows the default virtual environment provided by CAVEStudy. It consists of a virtual laboratory with a manipulation box where data can be inserted, and a board to present some text information.

\section{Applications}

To evaluate our approach of coupling a simulation and a virtual-reality environment, we implemented three different applications using CAVEStudy: Interactive Soccer, Diode Laser Simulation, and Molecular Dynamics. We use these

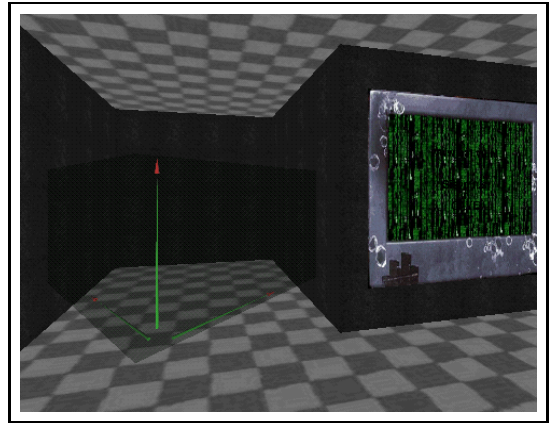

Figure 5. CAVEStudy laboratory

to illustrate the ease of incorporating an existing application, the usability of such a method, and the added value for the user. In the full paper, we give more insights on these applications.

\subsection{Interactive RoboCup}

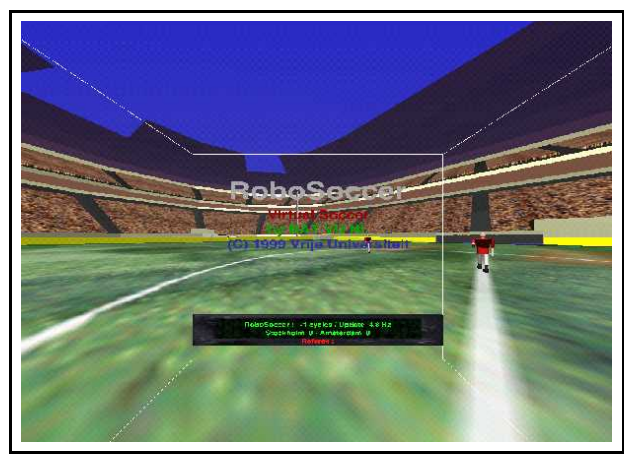

Figure 6. Interactive RoboCup

RoboCup (Robot Soccer) is a standard problem from Artificial Intelligence [7]. Its goal is to let teams of cooperating autonomous agents play a soccer match, using either real robots or simulated players. We constructed a VR environment in which humans in CAVEs at different geographic locations can play along with a running RoboCup simulation in a natural way. A central role is played by the so called Soccer Server, which keeps track of the state of the game and provides the players with information on the game. The players are individual processes that can request state information from the server and autonomously compute their behavior. The server also enforces the rules of RoboCup and ignores invalid commands from the players.

Our RoboCup VR system uses the unmodified existing server software. The players communicate with the server by sending soccer commands. The commands are expressed in a simple language, consisting of accelerations, 
turns, and kicks. The server discretizes time into slots and only the last command of a player within a time slot is executed. Also, the kick command requires the player to be close to the ball. We run such a system on a Myrinet cluster computer.

The CAVE program allows the user to be immersed in the game and to interact with it. We implemented a proxy which uses the same information and communication as the existing 2D visualization. The data set described in our CAVEStudy configuration file consists mainly of the player positions and the ball position. From successive states of the game, the visualization system computes several quantities such as direction, velocity and acceleration of the players. We built a virtual stadium (Figure 6) and a parameterized soccer player whose movements are interpolated between three different modes: standing still, walking, and running. We developed software to track the behavior of a human in the CAVE. One tracker is connected to the viewing glasses and monitors positional changes of the human player inside the CAVE. The second tracker is connected to the wand (a $3 \mathrm{D}$ mouse), which is used for global movements over the soccer field. The third tracker is attached to the foot of the human player and is used to recognize a kick. We convert tracker changes into soccer commands and transmit these to the server. Finally, we coupled two CAVEs located in Amsterdam and Stockholm [20]. The two CAVEs are connected to the same proxy, so the two humans participate in the same game.

The most difficult problem in realizing a virtual RoboCup system is caused by the latency of the simulation program. If the human player moves over the virtual soccer field, these moves happen almost instantaneously for the human. In contrast, the soccer server will require some time to process the change of position. Also, the wide-area (Internet) connection causes a substantial delay. This problem is a typical example of how a delay introduced by a simulation program can harm a natural and real-time interaction [15]. We are currently developing accurate and low-bandwidth algorithms for the navigation (walking across the soccer field) and the interaction (kicking detection) that generate commands to the server.

The first implementation of this system was done without CAVEStudy. We had to program the communication between the different components, which is tedious and error prone. Using CAVEStudy, the communication is automatically generated. More generally, the case study with RoboCup shows the applicability of CAVEStudy on the large class of agent/server systems.

\subsection{Diode laser simulation}

Another application we implemented is the visualization of a diode laser behavior, referred to as the Sisyphus Attrac-

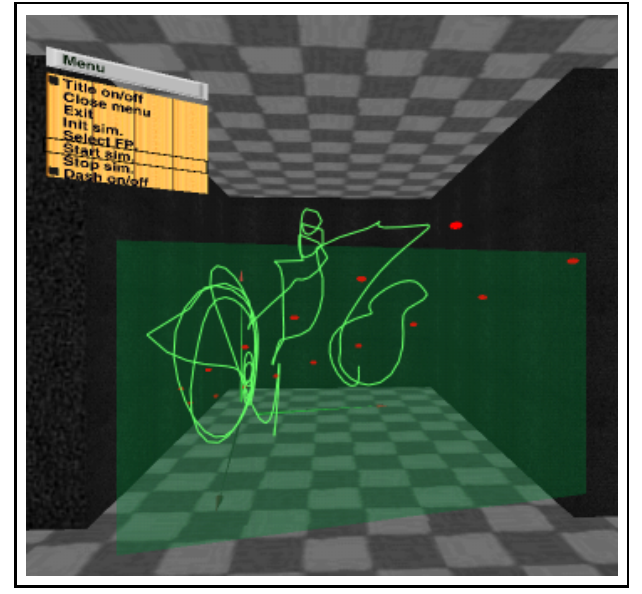

Figure 7. Diode laser simulation

tor [12]. Numerical simulations are performed for a semiconductor diode laser, subject to optical feedback. Due to the feedback, the resulting dynamical system has infinite degrees of freedom. The exploration and investigation of such a large data set calls for the immersion of the user into a representation of the parameter space. A simulation run generates a trajectory in such a space. In the $3 \mathrm{D}$ space provided by CAVEStudy, we decided to focus on the most natural phase space from the physical point of view (the output power, the inversion, and the phase difference) as shown in Figure 7.

A previous study [12] on the visualization of this simulation already gave a better insight into the dynamical behavior of the laser, but suffered severely from lack of interaction. With CAVEStudy, we linked the simulation running on an IBM SP2 to our CAVE. In a first step, the simulation computes some fixed points in the phase space for a given set of parameters. The user can interactively set the values of selected parameters using sliders. The fixed points serve as starting point of the simulation. These points are visualized, and the scientist can directly select one of these points to start the simulation. The computed trajectory is sent incrementally to the CAVE. The trajectory is visualized and can be manipulated by the scientist. The simulation can be stopped and re-started using a new starting fixed-point or different parameter values.

CAVEStudy's benefits are many-fold in this case; it is easier to use than a previous approach (batch-processing and offline visualization); the study of the initial-condition sensitivity of the laser is enhanced by the ability to modify the parameters of the simulation interactively; since our system does not require modifications of simulation code, we can deal very easy with the changes of a code still revised frequently; the interactive way in which physicists could test hypotheses and investigate the behavior of the diode 
laser helped them to gain a better insight in this complex system.

\subsection{Interactive Molecular Dynamics}

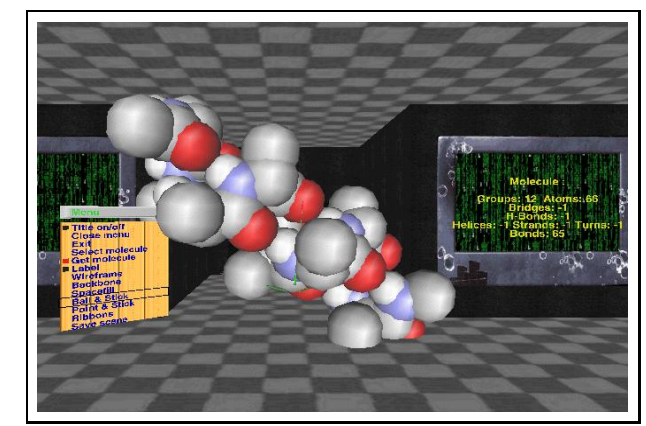

Figure 8. Interactive Molecular Dynamics

Our third application concerns the coupling of a molecular dynamics (MD) simulation to a virtual reality system. Molecular modeling tools are essential to design and study new molecules. For example, when steering a molecular dynamics simulation, the user can express external forces to help the system to overcome energy barriers, or can help in the search for likely geometric configurations in docking problems $[8,10]$. VR allows the scientist to gain a deeper understanding of the complex conformations in 3D. Moreover, modifying 3D structures or expressing forces is intrinsically a 3D process, for which the use of a immersive virtual environment is a perfect match.

As a feasibility study (and for later experiments on interaction and measurement), we wrapped the molecular dynamics NAMD [14] simulation and visualized it in a VR environment. Our current implementation allows a remote simulation running on the DAS parallel cluster computer [1] to be visualized in the CAVE. The input parameters we selected are the name of the molecule on which the simulation will be applied, the number of time steps of the simulation, and the temperature. It corresponds to the minimal set of parameters among the large possibilities of NAMD. We did not implement the interactive parameter selection yet, but parameters can be modified at starting time. As output of the simulation, we use the PDB description files produced as intermediate result during the execution. These files, which contain the position and velocity of all the atoms, are read by the proxy process and sent continuously to the visualization, showing the dynamic of the molecule. Several classic molecule representations are available. An example is shown in Figure 8.

The coupling of MD simulation to visualization has already been done before, but always by modifying source code. Using CAVEStudy, we were able to very quickly cou- ple NAMD to our virtual environment. With CAVEStudy, we can easily switch between several simulation packages. Furthermore, to steer such a simulation adequately, 3D forces should be expressed, which can efficiently be done in a 3D VR environment implemented by CAVEStudy.

\section{Conclusion}

In this paper, we described the CAVEStudy system that allows the scientist to interactively steer a simulation, without requiring any modification to the original program. It enables an interactive and immersive analysis of a simulation running on a remote computer.

A set of input, output, and graphical objects are specified by the scientist in a description file. These objects represent the input parameters of the simulation and the data produced. Using such a description, our system generates a server to control the simulation and to send data produced to the virtual environment. These data are used to update the graphical objects, which can be manipulated to steer the simulation. Thus, it is possible to visualize and control the program directly in the domain space of the simulation.

We run simulations on various systems, such as an IBM SP2 and a Myrinet cluster computer, and coupled them to several CAVE VR systems. Moreover, three real applications (soccer, laser simulation, molecular dynamics visualization) have been studied.

The lessons learned from these applications are that different types of applications can easily be implemented using CAVEStudy, that CAVEStudy is easy to use and to maintain compared to previous methods, and that $3 \mathrm{D}$ interactive visualization and steering help to gain a better insight in a complex system.

The whole CAVEStudy system will be available soon on our web site http://www.cs.vu.nl/ renambot/vr.

\section{References}

[1] H. Bal, R. Bhoedjang, R. Hofman, C. Jacobs, K. Langendoen, T. Rühl, and F. Kaashoek. Performance Evaluation of the Orca Shared Object System. ACM Transactions on Computer Systems, 16(1):1-40, Feb. 1998.

[2] C. Carlsson and O. Hagsand. DIVE - A Platform for Multi-User Virtual Environments. Computers and Graphics, 17(6):663-669, Nov.-Dec. 1993.

[3] A. Foster and C. Kesselman. The Grid: Blueprint for a New Computer Infrastructure. Morgan Kaufman, 1998.

[4] I. Foster and C. Kesselman. Globus: A Metacomputing Infrastructure Toolkit. The International Journal of Supercomputer Applications and High Performance Computing, 11(2):115-128, 1997.

[5] A. S. Grimshaw and W. A. Wulf. The Legion vision of a worldwide virtual computer. Communications of the ACM, 40(1):39-45, Jan. 1997. 
[6] D. Jablonowski, J. Bruner, B. Bliss, and R. Haber. VASE: The Visualization and Application Steering Environment. In Proceeding of Supercomputing'93, pages 560-569, 1993.

[7] H. Kitano, M. Veloso, P. Stone, M. Tambe, S. Coradeschi, E. Osawa, I. Noda, H. Matsubara, and M. Asada. The RoboCup Synthetic Agents Challenge 97. In M. Pollack, editor, 15th International Joint Conference on Artificial Intelligence, pages 24-29, 1997.

[8] J. Leech, J. F. Prins, and J. Hermans. SMD: Visual Steering of Molecular Dynamics for Protein Design. IEEE Computational Science \& Engineering, 3(4):38-45, Winter 1996.

[9] J. Leigh, A. Johnson, T. DeFanti, and M. Brown. A Review of Tele-Immersive Applications in the CAVE Research Network. In IEEE Virtual Reality'99, pages 180-187, 1999.

[10] D. Levine, M. Facello, P. Hallstrom, G. Reeder, B. Walenz, and F. Stevens. Stalk: An Interactive System for Virtual Molecular Docking. IEEE Computational Science, 4(2):5565, April-June 1997.

[11] M. R. Macedonia, M. J. Zyda, D. R. Pratt, P. T. Barham, and S. Zeswitz. NPSNET: A Network Software Architecture for Large-Scale Virtual Environment. Presence, 3(4):265-287, 1994.

[12] C. Mirasso, M. Mulder, H. Spoelder, and D. Lenstra. Visualization of the Sisyphus Attractor. Computers in Physics, 11(3):282-286, May/June 1997.

[13] J. Mulder, J. van Wijk, and R. van Liere. A Survey of Computational Steering Environments. Future Generation Computer Systems, 13(6), 1998.

[14] M. T. Nelson, W. F. Humphrey, A. Gursoy, A. Dalke, L. V. Kalé, R. D. Skeel, and K. Schulten. NAMD: A Parallel Object-Oriented Molecular Dynamics Program. The International Journal of Supercomputer Applications and High Performance Computing, 10(4):251-268, 1996.

[15] K. S. Park and R. Kenyon. Effects of Network Characteristics on Human Performance in a Collaborative Virtual Environment. In IEEE Virtual Reality'99, pages 104-111, 1999.

[16] S. Parker, M. Miller, C. Hansen, and C. Johnson. An Integrated Problem Solving Environment: the SCIrun Computational Steering System. In Hawaii International Conference of System Sciences, pages 147-156, Jan. 1998.

[17] C. Shaw, M. Green, J. Liang, and Y. Sun. Decoupled Simulation in Virtual Reality with the MR Toolkit. ACM Transactions on Information Systems, 11(3):287-317, July 1993.

[18] G. Singh, L. Serra, W. Png, and H. Ng. BrickNet: A Software Toolkit for Network-Based Virtual Worlds. Presence, 3(1):19-34, 1994.

[19] S. Singhal and M. Zyda. Networked Virtual Environments: Design and Implementation. Addison-Wesley, 1999.

[20] H. J. W. Spoelder, L. Renambot, D. Germans, H. E. Bal, and F. C. Groen. Man Multi-Agent Interaction in VR: a Case Study with RoboCup. In Poster Session - IEEE Virtual Reality'00, 2000.

[21] J. van Wijk and R. van Liere. An Environment for Computational Steering. Computer Society Press, 1997. 